\title{
Steen Hildebrandt \& Christian Waldstrøm 2006: Børsens Management Leksikon. 4. Ausgabe, 1. Auflage. København: Børsen, 592 Seiten, ISBN-10: 87-7664-081-7. ISBN-13: 978-87-7664-081-1, DKK 499,00.
}

Ob man es auf das Prinzip “Die anderen tun das auch” zurückführen kann, ob es auf einem Widerwillen gegen das Institut für Dänische Sprache (d.i. Dansk Sprognævn) beruht oder ob es einfach aus Unwissenheit resultiert, ist ungewiss und kann nicht zum Gegenstand dieser Rezension gemacht werden: Es ist jedenfalls eine Tatsache, dass immer mehr dänische Verlage in Buchtiteln Wörter auseinanderschreiben, die korrekt zusammengeschrieben werden sollten. Sogar das Rechtschreibwörterbuch Politikens Retskrivningsordbog (2001) stellt im Titel einen derart groben Fehler zur Schau, was, um es milde zu sagen, unakzeptabel, eigentlich aber nur peinlich ist (es hätte Politikens Retskrivningsordbog heißen müssen).

Rechtschreibung ist nichts, was missachtet werden darf. Schließlich handelt es sich um ein komplexes Phänomen mit tiefen Wurzeln und Verzweigungen in der allgemeinen Gesellschafts- und Kulturgeschichte sowie in der Sprachgeschichte, Sprachpädagogik, Sprachpolitik, Grammatik, Phonetik, Phonologie, Etymologie, Semantik, Syntax und Pragmatik (vgl. hierzu u.a. Kongsdal Jensen (1995) oder die respektiven Kapiteln in DSH). Rechtschreibung kann sogar mitunter von verhängnisvoller Bedeutung sein - z.B. in rechtlichen Zusammenhängen.

Gerade im Fall der Zusammenschreibung handelt es sich aber nicht nur um eine Frage der Rechtschreibung, sondern auch um eine Frage der Grammatik. Es ist eine Sache, ob jemand die Schreibweise “majonæse” der Variante “mayonnaise” vorzieht (oder umgekehrt). Beiden Formen steht grammatisch gesehen nichts im Wege - "management leksikon" oder "retskrivnings ordbog” können jedoch nur als inkorrekt bezeichnet werden (vgl. hierzu u.a. §§ 18 und 19 in Retskrivningsordbogen (2005) und § 97 in EDG).

Børsens Management Leksikon, hiernach BML, erschien zum ersten Mal 1984 beim Verlag Børsen. Diese Rezension bezieht sich auf die neue Ausgabe aus dem Jahr 2006. Der Klappentext verrät Folgendes über das Buch (hier vom Dänischen ins Deutsche übersetzt):

Neue und völlig überarbeitete Ausgabe des Børsens Management Leksikon. Das Lexikon enthält mehr als 3.000 Stichwörter und Begriffe. Die Herausgeber des Lexikons, Professor Steen Hildebrandt und Assistent Christian Waldstrøm, beide von der Universität Aarhus, haben mehr als ein Jahr dafür gebraucht, die wichtigsten Begriffe der Führung, Betriebsorganisation und Verwaltung zu finden und zu beschreiben. 
Børsens Management Leksikon ist ein einzigartiges und notwendiges Werkzeug für jede/n, der/die sich mit Fragen der Führung beschäftigt, sei dies praktisch als Leiter bzw. Manager oder Führungskraft auf mittlerer Ebene oder theoretisch als Forscher oder Student.

Es ist erfreulich, dass die beiden Herausgeber, die zugleich Verfasser des Werkes sind, auf der letzten Seite des Buches mit biografischen Informationen vorgestellt werden. Herausgeber- und/oder Verfasserinformationen fehlen oft in Wörterbüchern und Lexika, obwohl sie wichtig sind, nicht nur um die Neugierde eifriger Rezensenten zu befriedigen, sondern vor allem, weil sie das jeweilige Werk legitimieren und ihm Autorität verleihen.

BML ist ein dickes, solide gebundenes und schönes Buch. Das kohlschwarze Vorsatzblatt ist z.B. ein sehr schönes Detail. Gute Ausstattung und äußere Gelungenheit sind leider keine Selbstverständlichkeit und die Herausgeber und der Verlag verdienen dafür Lob und Dank. Ein großes Minus in diesem Zusammenhang ist jedoch der Flattersatz, der sich durch das ganze zweispaltig gesetzte Werk zieht: Er ist der Zugriffsstruktur nicht förderlich. Ein fester Satz bringt eine gewisse Ruhe in die Textmasse, etwas, was in einem Werk wie dem BML, das ohnehin ein recht luftiges Layout aufweist, desto notwendiger gewesen wäre.

Doch was enthält eigentlich ein Wörterbuch des Managements? Es ist schwer, den Unterschied zwischen den Artikelbeständen zu erkennen, wenn man das Buch mit einem gewöhnlichen Lexikon der Betriebswirtschaftslehre vergleicht. Dies ist aber nicht als Kritik aufzufassen, sondern nur als Antwort auf eine Frage, die wahrscheinlich vielen Lesern dieser Rezension sonst unbeantwortet bleiben würde. Management ist die Kunst, die humanen wie auch die pekuniären Ressourcen eines gegebenen Betriebs zu verwalten und imstande zu sein, die auftretenden Herausforderungen zu bewältigen und die gesetzten Ziele des Betriebs zu erreichen. Der Markt ist ein Kampfplatz, um nicht zu sagen ein Schlachtfeld, mit komplexen Spielregeln, wo es entscheidend ist, wer die Mitspieler sind und welche Werkzeuge man zur Verfügung hat, um das Spiel so erfolgreich wie möglich meistern zu können. Die Verfasser des Wörterbuches möchten den Wörterbuchbenutzern zeigen, welche wissenschaftlichen Werkzeuge man anwenden kann, um gute Ergebnisse im Unternehmensbereich zu erzielen. Dabei geht es den Verfassern nicht nur darum, traditionelles BWL-Wissen enzyklopädisch darzubieten, sondern auch Brücken zu anderen Fachdisziplinen zu schlagen. Neben traditioneller Führungstheorie einerseits (vgl. Arbejderkollektivet, Bruttonationalprodukt, Eksportstøtte, Infrastruktur) und neueren Begriffen andererseits (vgl. Corporate social responsibility, Entreprenør, Innovationsbeslutningsmodellen, Virksomhedsetik) versucht BML, Altes und Neues in Sachen Führung und Betriebsorganisationslehre zu 
vereinigen, wobei die Auswahl der beschriebenen Themen gleichzeitig interdisziplinär angelegt ist.

Mit 3.000 Artikeln ist der Artikelbestand relativ groß, die Auswahl jedoch nicht unproblematisch. Es wurden viele Artikel aufgenommen, die trotz der erwähnten Interdisziplinarität in so einem Buch besser keine Berücksichtigung gefunden hätten. Es ist durchaus möglich, dass man problemlos für die Aufnahme der vielen technici termini aus der Philosophie und Psychologie argumentieren kann, kaum zu verteidigen ist jedoch die Aufnahme folgender Artikel: Antropologi, Etnografi, Etnologi, Firewall, Fysikalisme, Kriterium, Layout, Lingvistik, Paradigme, Semantik, Semiotik, Story board, Strukturalisme, Tautologi, Teknologi, Tekstbehandling, Teleologi, Teorem, Utopi, Videnskabssociologi und Æstetik.

Wie lässt sich diese Auswahl erklären? Handelt es sich hier um Nachlässigkeit? Mangel an Überlegungen? Oder handelt es sich um eine bewusste Wahl? Falls die Auswahl tatsächlich bewusst ist, wie ist sie (wissenschaftlich bzw. lexikographisch) zu verantworten? Viele glauben, je mehr Stichworte ein Wörterbuch enthält, desto besser. Folglich kann man sich - so könnte argumentiert werden - über diese breite Auswahl nicht beschweren. Grundsätzlich sind solche Gedanken nicht falsch, eher unrealistisch und dazu noch metalexikographisch gesehen problematisch. Das Problem besteht darin, dass kein Wörterbuch alle Wörter enthalten kann, dazu gibt es einfach zu viele Wörter in der Sprache. Sogar ein Werk wie das 33-bändige Ordbog over det danske Sprog (das dänische Pendant zum Grimmschen Wörterbuch) enthält bei Weitem nicht alle Wörter der dänischen Sprache. Die Aufnahme einer beliebigen Wortmasse in einem gegebenen Wörterbuch bedeutet immer, dass andere, vielleicht relevantere Wörter nicht aufgenommen bzw. nicht ausreichend behandelt werden können. Der primäre Gegenstandsbereich des BML ist das Management bzw. die Betriebsorganisationslehre und vor allem darauf sollte sich das Werk konzentrieren - natürlich abgesehen von Nachbardisziplinen bzw. benachbarten Fächern. Sind die Verfasser des Werkes wirklich der Auffassung, dass der primäre Gegenstandsbereich möglichst erschöpfend behandelt worden ist? Dazu kommt noch, dass sich die Verfasser aufs Glatteis begeben, denn sie sind nicht etwa Anthropologen, Linguisten, Philosophen, Semiotiker usw., sondern Betriebswirte bzw. Fachmänner im Bereich Wirtschafts- und Betriebsorganisationslehre (vgl. die Biografien auf der letzten Seite des Buches). Schließlich - und dies ist vielleicht am wichtigsten - kollidiert ein solches Verfahren, d.h. die Breite der Auswahl, mit einem grundlegenden Prinzip, dessen Wichtigkeit in der modernen Metalexikographie wohl kaum mehr in Frage gestellt wird: Das Prinzip der Funktionalität. Welche Funktionen hat das BML? Welche Benutzerbedürfnisse sollen befriedigt werden?

In der modernen lexikographischen Funktionslehre sind folgende lexiko- 
graphische Funktionen, welche die vorausgesehenen Benutzerbedürfnisse berücksichtigen sollen, als zentral aufzufassen (vgl. Bergenholtz/Tarp (2002; 2003; 2005) und Tarp (1992; 2006)):

- $\quad$ kommunikative Funktionen: Hilfe bei Textproduktion, Hilfe bei Textrezeption, Hilfe bei Übersetzung

- $\quad$ kognitive Funktionen: Hilfe bei systematischem Wissensbedarf, Hilfe bei sporadischem Wissensbedarf, ...

Mit kommunikativen Benutzerbedürfnissen sind Situationen gemeint, in denen der Benutzer ein Problem lösen möchte, das im Zusammenhang mit der Produktion, Rezeption oder Übersetzung eines Textes entstanden ist. Mit kognitiven Benutzerbedürfnissen sind Situationen gemeint, in denen der Benutzer den Bedarf hat, konkretes oder systematisches Wissen über irgendetwas - sei es ein sprachliches oder nicht-sprachliches Phänomen - zu erwerben.

Über lexikographische Funktionen oder Benutzerbedürfnisse wird im BML nichts Explizites gesagt, es ist jedoch deutlich, dass das Werk primär zur Wissensaneignung konzipiert wurde, d.h. es handelt sich um ein kognitiv-funktionales Wörterbuch. Die kommunikative Benutzersituation Textproduktion wird in dem Werk nicht vorausgesehen, was u.a. dadurch zum Ausdruck kommt, dass sprachliche Informationen (z.B. Angabe von Genus, Flexion und Wortklassenzugehörigkeit) gänzlich fehlen. Gerade in Hinsicht auf die Textproduktion erweist sich auch die irreführende Ortographie als unzweckmäßig: Sämtliche Lemmata sind mit großen Anfangsbuchstaben geschrieben. Dies ist eine lexikographische Unsitte, ein pädagogischer Fehlgriff und ein ortographisches Verbrechen. Der Wörterbuchbenutzer sieht sich mit dem Problem konfrontiert, nicht zu wissen, ob ein Schlagwort tatsächlich mit großem oder kleinem Anfangsbuchstaben geschrieben werden muss. Ist z.B. Corporate Social Responsibility als ein Proprium aufzufassen und demgemäß korrekt mit großen Anfangsbuchstaben geschrieben? Falls ja, muss man dann Corporate Social Responsibility oder kann man einfach Corporate social responsibility schreiben? Falls nein, heißt es wohl corporate social responsibility?

Die kommunikative lexikographische Funktion Hilfe bei der Textrezeption wird hingegen berücksichtigt, indem das Werk recht gründliche und mitunter ausführliche Bedeutungserklärungen bzw. Darlegungen des gegebenen Wortes bzw. Sachverhaltes anbietet.

Die Funktion Hilfe bei Wissensaneignung hätte verbessert werden können, wenn weit mehr biografische Artikel Aufnahme gefunden hätten. Wieso sind im ganzen Werk nur (in invertierter Form) Ichak Adizes, Erik Johnsen, Michael Porter und Edgar H. Schein aufgenommen? Man hätte genauso gut auf biografische Artikel verzichten können. Auf jeden Fall ist es außergewöhnlich für 
ein Lexikon, das biografische Artikel enthält, nur vier oder fünf solcher Artikel anzubieten. Oder gibt es etwa nur so wenige tonangebende Persönlichkeiten in der Welt der Betriebsorganisationslehre?

Das Werk fokussiert auf Entwicklungstheorien und Werkzeuge im Zusammenhang mit dem Management und verzichtet darauf, Begriffe zu behandeln, die mit Arbeitsintensität und Leistungsfähigkeit seitens der Arbeitnehmer oder Arbeitgeber zu tun haben. Krisenführungstheorie (crisis management theory) wird ebenso nicht behandelt. Kann dies daran liegen, dass das Buch in einer Zeit erschienen ist, in der sich die dänische Gesellschaft in einer wirtschaftlichen Hochkonjunktur befindet und in materiellem Reichtum schwelgt?

Es wäre nützlich gewesen, wenn das Buch sich stärker auf aktuelle Themen konzentriert hätte, z.B. Management im Zusammenhang mit interner und externer Kommunikation, und wenn es darüber hinaus visionärer angelegt wäre, was die Nutzung menschlicher Ressourcen und deren strategische Entwicklung betrifft. Vgl. zu Letzterem z.B. die Artikel Personaleudvcelgelse und Personkapital, die viel ausführlicher hätten ausfallen können.

Es wäre bei Weitem visionärer gewesen, den Begriff Firewall mit der Frage zu verbinden, wie in einem Betrieb Sicherheitsbarrieren auf mehreren Ebenen etabliert werden. In diesem Zusammenhang wäre es auch interessant gewesen, Artikel über Optionen wie Videoüberwachungssysteme als Teil eines internen Handlungsplans aufzunehmen. Wir wären erfreut, wenn in einer nächsten Auflage des Buches Themen wie (Aspekte der) Sicherheit und (neue) Gefahren für das Geschäft, das Prestige und die Existenz eines Betriebes ausführlicher behandelt werden könnten (z.B. in Zusammenhang mit Terrorismus und neuen Formen der Industriespionage).

In einer neuen Auflage könnte man des Weiteren einige zusätzliche Artikel über die Europäische Union hinzufügen - u.a. darüber, wie man sich in dieser wechselhaften Welt des Kapitals die Arbeitsform(en) und Prinzipien der Union zunutze machen kann.

Auch die internetbasierten Technologien kommen im Werk zu kurz, vgl. z.B. IT und Kredsløbskort. Eine ausführlichere Behandlung erfahren $\boldsymbol{E}$-laring und Internethandel (vgl. $\boldsymbol{E}$-business und $\boldsymbol{E}$-handel), allerdings wird $\boldsymbol{E}$-work erklärt, ohne dass die Konsequenzen für verschiedene Gruppen von Arbeitnehmern erwähnt werden.

Artikel wie Enkeltmandsfirma können als Versuch aufgefasst werden, Jungunternehmer zu berücksichtigen. Angebrachter wäre es jedoch gewesen, wenn die Verfasser potentielle Jungunternehmer auch über die versteckten Kosten eines neugegründeten Unternehmens und die unerwarteten ökonomischen Probleme, die damit verbunden sind, gewarnt hätten. Lobenswert ist allerdings, dass die Verfasser auf interne und externe Strategien, die für Jungunternehmer 


\section{2}

von Interesse sein könnten, aufmerksam machen.

Wenn überhaupt rechtliche und organisatorische Sachverhalte miteinbezogen werden müssen, wäre es wünschenswert gewesen, den Wörterbuchbenutzern etwas mehr Aufklärung über das Gesellschaftsrecht anzubieten, wie zum Beispiel über die Haftpflicht gegenüber Kreditoren, Haftpflicht gegenüber existierenden und austretenden Akteuren, die Befriedigungsgrundlage der Gesellschaftskreditoren und wesentliche Unterschiede zwischen Aktien- und anderen Gesellschaftsformen, um nur einige Bespiele zu nennen.

Im Großen und Ganzen ist das Wörterbuch jedoch durchaus hilfreich und bietet eine spannende Lektüre für diejenigen, die sich für die Welt der Wirtschaftsorganisationen interessieren. ${ }^{1}$

\section{Bibliographie}

Bergenholtz, Henning/Tarp, Sven 2002: Die moderne lexikographische Funktionslehre. Diskussionsbeitrag zu neuen und alten Paradigmen, die Wörterbücher als Gebrauchsgegenstände verstehen. In Lexicographica 18, 253-263.

Bergenholtz, Henning/Tarp, Sven 2003: Two opposing theories: On H.E. Wiegand's recent discovery of lexicographic functions. In Hermes 31, 171-196.

Bergenholtz, Henning/Tarp, Sven 2005: Wörterbuchfunktionen. In Barz, Irmhild/ Bergenholtz, Henning/Korhonen, Jarmo (Hrsgg.): Schreiben, Verstehen, Übersetzen und Lernen : Zu ein- und zweisprachigen Wörterbüchern mit Deutsch. Frankfurt am Main/Bern/New York/Paris: Peter Lang, 11-25.

DSH = Peter Skautrup: Det danske Sprogs Historie I-V. Udgivet af Det Danske Sprogog Litteraturselskab. København: Gyldendal 1944-1970.

EDG = Paul Diderichsen: Elementcer Dansk Grammatik. 3. udgave, 2. oplag. København: Gyldendal 1966.

Kongsdal Jensen, Ole 1995: Den franske ortografi. København: Museum Tusculanum.

Retskrivningsordbogen. Udgivet af Dansk Sprognævn. 3. udgave, 4. oplag med ændrede kommaregler. København: Alinea/Aschehoug 2005.

Tarp, Sven 1992: Prolegomena til teknisk ordbog. Århus: Institut for Spansk, Handelshøjskolen i Århus.

Tarp, Sven 2006: Leksikografien i grcenselandet mellem viden og ikke-viden. Generel leksikografisk teori med scerligt henblik på lørnerleksikografi I-II. Århus: Center for Leksikografi.

Loránd-Levente Pálfi \& Spiros Divaris Vesterdahl

1 Wir danken Uwe Mayer und Anja Frentzel für die kritische Durchsicht einer früheren Version dieser Rezension. 